\title{
Evaluation de la diversité floristique et estimation du taux de séquestration de carbone des arbres en alignement de voies de la commune de Daloa (Côte d'Ivoire)
}

\author{
Justin Kanga KOUASSI ${ }^{1}$, Henri Kouadio KOUASSI ${ }^{2}$ et Hervé Roland KOUASSI ${ }^{3}$ \\ ${ }^{l}$ Doctorant à l'Université Jean Lorougnon Guédé de Daloa, Unité de Formation et de Recherche en \\ Environnement, BP 150 Daloa, Côte d'Ivoire. \\ ${ }^{2}$ Laboratoire de Biologie et Ecologie végétales, UFR/Agroforesterie, Université Jean Lorougnon Guédé, BP \\ 150 Daloa, Côte d'Ivoire. \\ ${ }^{3}$ Département des Sciences et Technologie, Section Sciences de la Vie et de la Terre, Ecole Normale \\ Supérieure, Abidjan, 08 BP 10 Abidjan 08, Côte d'Ivoire. \\ *Auteur correspondant ; E-mail : kouassikangajustin@ gmail.com; Tel: +225 57103899 ; 46448483.
}

\section{RESUME}

Aujourd'hui, l'arbre en milieu urbain est devenu un élément essentiel pour un environnement urbain vivable et durable. Cependant, dans nos villes africaines l'accent n'est pas mis sur ce patrimoine forestier. Cette étude a été a été réalisé dans le but de déterminer la diversité floristique et d'évaluer les services écosystémiques fournis par les arbres en alignement de voies de la ville de Daloa. Pour ce faire, les voies bitumées ont été choisies et la méthode d'inventaire itinérante a été utilisée. En plus de noter la présence ou l'absence de tous les arbres, ceux ayant un DBH $\geq 10 \mathrm{~cm}$ et une hauteur $\geq 2 \mathrm{~m}$ ont été estimés. Ces inventaires ont permis de recenser 224 individus repartis en 19 espèces, 16 genres et 14 familles. Parmi ces espèces, les espèces introduites sont les plus dominantes avec 9 espèces. Le genre Ficus est le plus représenté avec quatre (4) espèces. En outre, seulement 6,15 Km de voie sont encore bordés d'arbre pour une superficie de 9,41 ha. Le taux de carbone séquestré par l'ensemble des arbres s'élève à 42,92 tonnes d'où l'intérêt de les maintenir et de renforcer leurs densités et leurs diversités pour un équilibre écologique urbain plus durable.

(c) 2018 International Formulae Group. All rights reserved.

Mots clés: Diversité floristique, Services écosystémique, Arbres d'alignement, Daloa, Côte d'Ivoire.

\section{Evaluation of the floristic diversity and estimation of the rate of carbon sequestration of trees of alignment of the municipality of Daloa (Côte d'Ivoire)}

\begin{abstract}
Today, urban tree has become an essential element for a livable and sustainable urban environment. However, in African cities the emphasis is not on this forest heritage. This study was conducted to determine floristic diversity and to evaluate the ecosystem services provided by trees of alignment of the city of Daloa. To do this, asphalt roads were chosen and the itinerant method was used. In addition to noting the presence or absence of all trees, those with a DBH $\geq 10 \mathrm{~cm}$ and a height $\geq 2 \mathrm{~m}$ were estimated. These inventories allowed to identify 224 individuals distributed among 19 species, 16 genera and 14 families. Among these species,
\end{abstract}


those introduced are the most dominant with 9 species. The genus of Ficus is the most represented with four (4) species. In addition, only $6.15 \mathrm{~km}$ of track are still tree lined for an area of 9.41 ha. The carbon content sequestered by all the trees amounts to 42.92 tonnes, hence the interest of maintaining them and reinforcing their densities and diversities for a more sustainable urban ecological balance.

(C) 2018 International Formulae Group. All rights reserved.

Keywords: Flora diversity, Ecosystem services, Trees of alignment, Daloa, Côte d'Ivoire.

\section{INTRODUCTION}

Les villes ivoiriennes en général et celles situées en zone forestière en particulier ont toujours été fortement marquées par une croissance urbaine accélérée et non maîtrisée. Ce phénomène a amplifié et accéléré la réorganisation de l'espace urbain et a fortement contribué à accentuer les effets néfastes sur l'environnement. Les impacts environnementaux et sociaux qui en résultent, risquent de s'amplifier dans les années à venir (Vrain, 2003). Or, parmi les stratégies pouvant apporter une contribution à la résolution de ces problèmes, la foresterie urbaine suscite de nos jours de plus en plus d'intérêt par la diversité de ses fonctions. En effet, La végétation urbaine remplit des fonctions écologiques essentielles et assez importante dans l'équilibre urbain dans le contexte actuel des changements climatiques drastique (Osseni et al., 2015). Ces espaces sont des moyens de conservation des végétaux et d'amélioration du bien-être des citadins.

Bien que la société urbaine ait toujours exprimé le besoin d'avoir des espaces verts (Reygrobellet, 2007), la ville de Daloa sur laquelle se focalise notre étude souffre d'une pénurie d'espaces verts en général et d'arbres en alignement de voie en particulier. Par ailleurs, aucune étude n'a été réalisée jusqu'à ce jour en matière de foresterie urbaine dans la commune même si cette thématique reste peu développée dans la recherche scientifique en Côte d'Ivoire. Or la connaissance de la foresterie urbaine permet d'optimiser les méthodes d'aménagement et de gestion des arbres et des espaces verts urbains (Polorigni et al., 2014) qui répond à des enjeux non seulement sociaux et écologiques mais aussi économiques.
Face à une telle situation, il est nécessaire de susciter, à travers les résultats des études, l'éveil de la conscience environnementale chez les populations urbaines. La présente étude s'est fixée pour objectif général d'évaluer la diversité des arbres en alignement de voies de la ville de Daloa et leur rôle dans l'amélioration du bienêtre des populations à travers les services écosystémiques fournis par celles-ci. Plus spécifiquement, il s'est agi d'évaluer la diversité des arbres plantés en alignement des voies et à estimer la biomasse totale afin d'en déduire la quantité de carbone pouvant être séquestrée par ces arbres.

\section{MATERIEL ET METHODES Sites de l'étude}

Chef lieux de la région du HautSassandra, la ville de Daloa est localisée entre $6^{\circ} 30$ et $8^{\circ}$ de latitude nord et entre $5^{\circ}$ et $8^{\circ}$ de longitude ouest. Elle couvre une superficie de 9 650,75 ha (Figure 1). Caractérisée par une forte dynamique démographique et une extension spatiale sans précédent, Daloa représente le principal pôle urbain et le pôle économique majeur du centre-ouest ivoirien. La commune jouit d'un climat de type tropical humide avec une précipitation moyenne annuelle comprise entre $1200 \mathrm{~mm}$ et $1600 \mathrm{~mm}$ par an (Kouamé et al., 2015) et une température moyenne annuelle de $26{ }^{\circ} \mathrm{C}$ (Tra Bi et al., 2015). Les sols de la municipalité sont ferralitiques moyennement à fortement désaturés et le type de forêt spécifique est la forêt dense humide semi-décidue à Celtis spp et Triplochiton scleroxylon (Guillaumet et Adjanohoun, 1971). Mais en plus des causes de dégradation traditionnellement connues, l'urbanisation grandissante de la commune constitue actuellement l'un des facteurs 
important de modification de ces écosystèmes. Pourtant, les arbres en alignement de voies qui sont sensés compenser en partie cette perte énorme sont presqu'inexistant dans la ville.

\section{Collecte de données floristiques}

La méthode d'inventaire itinérante a été utilisée pour mener cette étude. Pour ce faire, tous les arbres en bordures des voies bitumées ont été recensés. Le choix a été porté sur ces voies parce qu'elles constituent les seules voies bordées d'arbre en alignement de la ville. En parcourant ces voies, les espèces d'arbres présentes en bordures des voies ont été identifiées et des mesures de diamètre et de hauteur ont été réalisées sur ceux ayant un diamètre supérieur ou égal à $10 \mathrm{~cm}$ et une hauteur supérieure ou égale à $2 \mathrm{~m}$. Les mensurations concernaient le diamètre à hauteur de poitrine (DBH). La largeur considérée était la distance séparant 2 arbres opposés.

\section{Analyse de la flore \\ Diversité qualitative}

Pour chaque voie inventoriée, le nombre d'espèces, de genres et de familles des taxons a d'abord été déterminé selon la classification APG IV (2016). Les types morphologiques et les chorologies sont assignés à Aké Assi $(2001,2002)$ et Raunkier (1984). Ces paramètres ont permis d'avoir une idée globale de la diversité qualitative de la flore étudiée.

\section{Diversité quantitative}

Elle a été évaluée par les indices de diversité couramment utilisés dans les études floristiques à travers le monde. Il s'agit de l'indice de diversité de Shannon-Weaver $(\mathrm{H})$, de Simpson (S) et de Pielou (E). Ces différents indices ont été calculés grâce au logiciel R 2.8.0. Afin de caractériser le degré de ressemblance des listes d'espèces des différentes voies, le coefficient de similitude a été calculé. Il est obtenu suivant l'équation :

\section{Cs $(s)=100 \times 2 c /(a+b)$}

Avec $\mathrm{a}=$ nombre d'espèces du milieu $\mathrm{A} ; \mathrm{b}=$ nombre d'espèces du milieu $\mathrm{B}, \mathrm{c}=$ nombre d'espèces communes aux deux milieux écologiques. Les valeurs $(\mathrm{Cs})$ varient de 0 à $100 \%$ selon que les deux sites sont de compositions floristiques totalement différentes $(\mathrm{c}=0)$ ou identiques $(\mathrm{a}=\mathrm{b}=\mathrm{c})$.

Diversité structurale des espèces ligneuses

La diversité structurale de chaque voie a été caractérisée à partir de la densité des arbres, de l'estimation de la surface terrière des taxons de chaque zone investiguée et de la distribution des tiges dans les classes de diamètre. La densité qui désigne le nombre d'individus, d'espèces par unité de surface dans une formation végétale a pour expression mathématique :

$$
\mathbf{D}=\mathbf{n} / \mathbf{s}
$$

Avec $\mathrm{D}=$ la densité $; \mathrm{n}=$ nombre de tiges dans le milieu considéré et $s=$ surface totale du milieu en ha. Elle est exprimée en tiges/ha.

La surface terrière qui est la somme de la surface des sections transversales des troncs de tous les arbres d'un relevé à $1,30 \mathrm{~m}$ audessus du sol (Rondeux, 1993) a également été estimé grâce à la formule : Elle s'exprime en $\mathrm{m}^{2}$.

$$
\mathrm{G}=\sum \pi D^{2} / 4
$$

\section{Estimation de la biomasse aérienne et du taux de carbone séquestré}

Les quantités de biomasse aérienne des différents individus ont été déterminées à l'aide des équations allométriques générales des arbres urbains ou Urban General Equations (Aguaron et McPherson, 2012) qui calculent la biomasse totale. Le développement et l'application de l'équation générale est une approche pour résoudre les nombreuses variations, incertaines et lacunes liées aux équations allométriques spécifiques relatives à la biomasse des arbres ou des arbustes pour les forêts urbains (McHale et al., 2009). Des équations spécifiques sont appliquées selon le type d'arbre en présence (arbres urbains, palmier à huile, rônier et cocotier). Ainsi, ces différentes équations s'écrivent comme suit :

$$
\begin{gathered}
\text { Biom }(t)(\text { arbres urbains })= \\
0,16155 \times \mathrm{DBH}^{2,310647}
\end{gathered}
$$


Biom (t) (palmier à huile, rônier et cocotier) = $1,282 \times(7,7 \mathrm{H}+4,5)$

Dans ces formules, Biom ( $\mathrm{t}$ ) désigne la biomasse totale exprimée en $\mathrm{kg} /$ tige ; $\mathrm{H}$ la hauteur totale de l'arbre mesurée en mètre et DBH désigne le diamètre à hauteur de poitrine exprimé en centimètre. Ensuite le taux de carbone séquestré est obtenu en appliquant un facteur de conversion noté $\mathrm{CF}$ à la biomasse totale. Ce facteur est de 0,5 (Malhi et al., 2004). Enfin, le dioxyde de carbone $\left(\mathrm{CO}_{2}\right)$ séquestré est obtenu en faisant intervenir le rapport des masses molaires du carbone et du $\mathrm{CO}_{2}$. Cela revient à multiplier le taux de carbone séquestré par 0,27 .

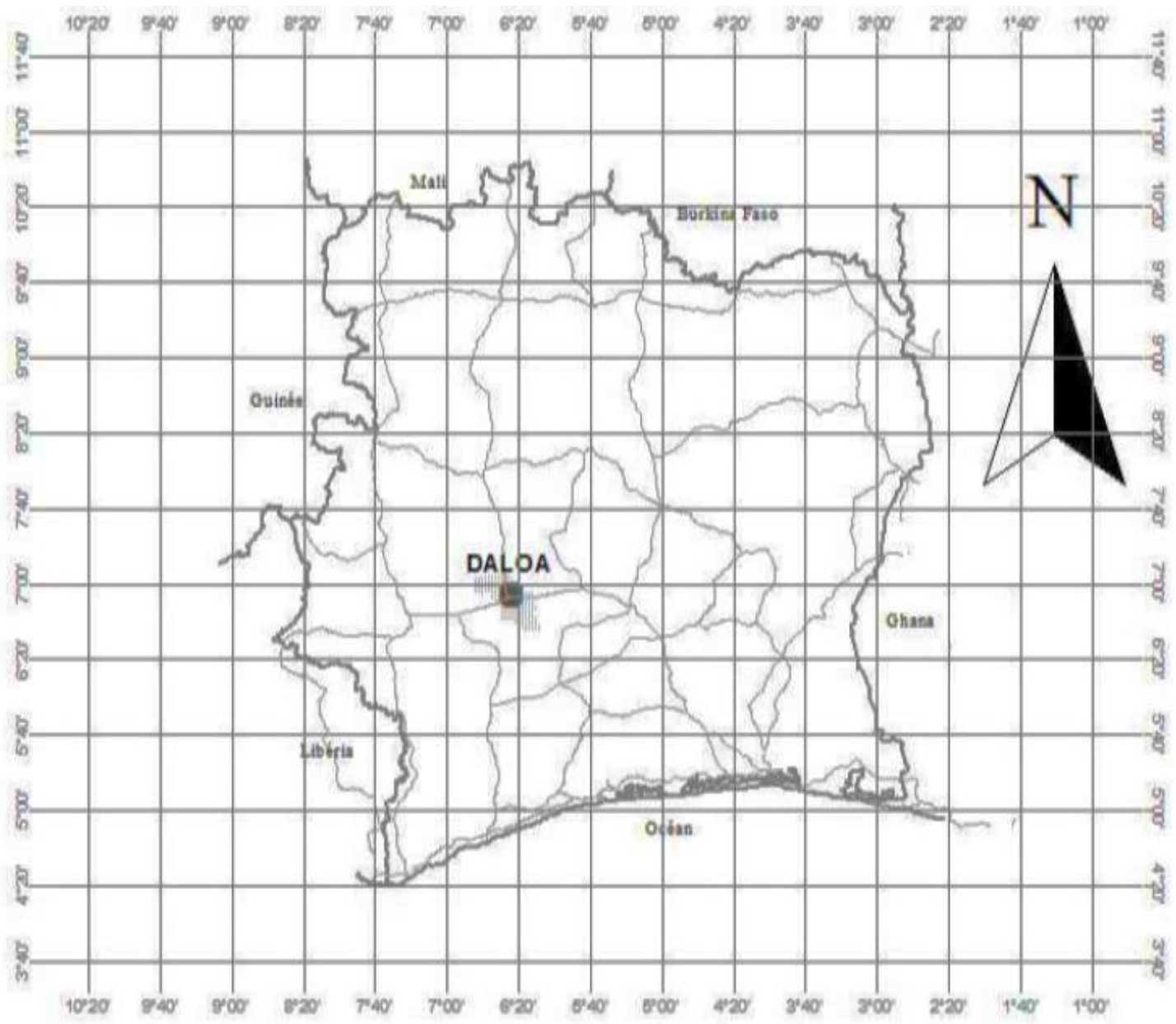

\section{Roads network}

100

Site

Department of Daloa

Figure 1: Localisation du site d'étude. 


\section{RESULTATS}

\section{Richesse et composition floristiques des arbres en alignement de voies}

Sur l'ensemble des axes routiers bitumés de la ville de Daloa, seulement sept (7) voies comportent encore des arbres en alignement. La flore ligneuse de l'ensemble de ces voies s'élève à 301 individus. Cependant, les ligneux de DBH $\geq 10 \mathrm{~cm}$ et de hauteur $\geq 2 \mathrm{~m}$ sont de 224 individus et se répartissent en 19 espèces, 16 genres et 14 familles (Tableau 1). La composition et la richesse spécifique de la flore inventoriée varient d'une voie à une autre. La typologie des arbres en alignement varie d'une voie à une autre. Cependant les arbres ayant une grande fréquence de rencontre sont Ficus benjamina $(0,86)$ suivie de Mangifera indica et de Senna siamea $(0,71)$. Enfin nous avons Azardirachta indica et Ficus polita $(0,57)$. Le genre le plus représenté en nombre d'espèces est Ficus (4 espèces). Tous les autres genres sont représentés par une seule espèce. Les espèces comptant le plus grand nombre d'individu par ordre décroissant sont Polyalthia logifolia (46 individus), Terminalia mentaly (38 individus) et Ficus benjamina (34 individus). L'analyse des familles, quant à elle, indique que les familles les plus représentées en nombre d'espèces sont les Moraceae $(21,43 \%)$ suivie des Annonaceae $(20,54 \%)$ et enfin des Combretaceae $(16,96 \%)$. Dans la flore de l'étude, les types biologiques les plus représentatives sont les Microphanérophytes (15 espèces). Les Mésophanérophytes et les Nanophanérophytes sont représentés respectivement par 3 et 1 espèce. La détermination de la chorologie de la flore étudiée a révélé une abondance des espèces introduites.

\section{Diversité des arbres en fonction des voies}

Pour l'ensemble des voies, les valeurs des indices de Shannon, d'Equitabilité et de Simpson estimées sont respectivement de l'ordre de 2,$74 ; 0,95$ et 0,95 .
Par ailleurs le Tableau 2 présente le degré de ressemblance entre les différentes voies. Les estimations de la similarité entre les espèces qui bordent les voies ont donnés des valeurs très faibles dans leur ensemble (Tableau 2). Ces valeurs indiquent une forte dissemblance en termes d'arbres en alignement entre les voies de la commune de Daloa. Cependant, la comparaison 2 à 2 entre les différents biotopes indique que seul les voies Boulevard et Rondpoint-Parvis ; ParvisAcémont et BCEAO-Feu Soleil 1 et la voie BHCI-carrefour Allocodrome présentent une ressemblance floristique.

\section{Structure horizontale de la végétation}

La distribution des arbres dans les classes de diamètre révèle une inégale répartition de ceux-ci. La classe de diamètre [10 - $20 \mathrm{~cm}$ [renferme le plus grand nombre d'individu (59) mais présente l'une des plus faible surface terrière. Au même moment, la classe de diamètre $[90-100 \mathrm{~cm}$ [avec seulement 2 individus, couvre $1,29 \mathrm{~m}^{2}$. La somme des surfaces terrières de l'ensemble des arbres est de 41,19 $\mathrm{m}^{2}$ (Tableau 3).

De toutes les voies, la plus dense est le boulevard. Celle-ci regorge 80,06 tiges/ha. Elle se distingue énormément de toutes les autres (Figure 2). La plus faible densité est observée sur les voies Rondpoint-Parvis et Parvis-Acémont (Tableau 4).

\section{Estimation de la biomasse ligneuse totale et du taux de carbone séquestré}

Cette étude révèle que la teneur en biomasse aérienne sèche de l'ensemble des arbres d'alignement de la ville de Daloa est de 317,89 tonnes et le taux de carbone qui en résulte est de 158,96 tonnes. Le taux de carbone séquestré correspondant s'élève à 42,92 tonnes. Ce taux est le plus élevé sur la voie Monument-grande mosquée et Bhcicarrefour allocodrome tandis qu'il est plus faible sur les voies Rondpoint-Parvis et Parvis-Acémont (Tableau 4). 
Tableau 1: Estimation de la richesse et composition floristique de la flore de l'étude.

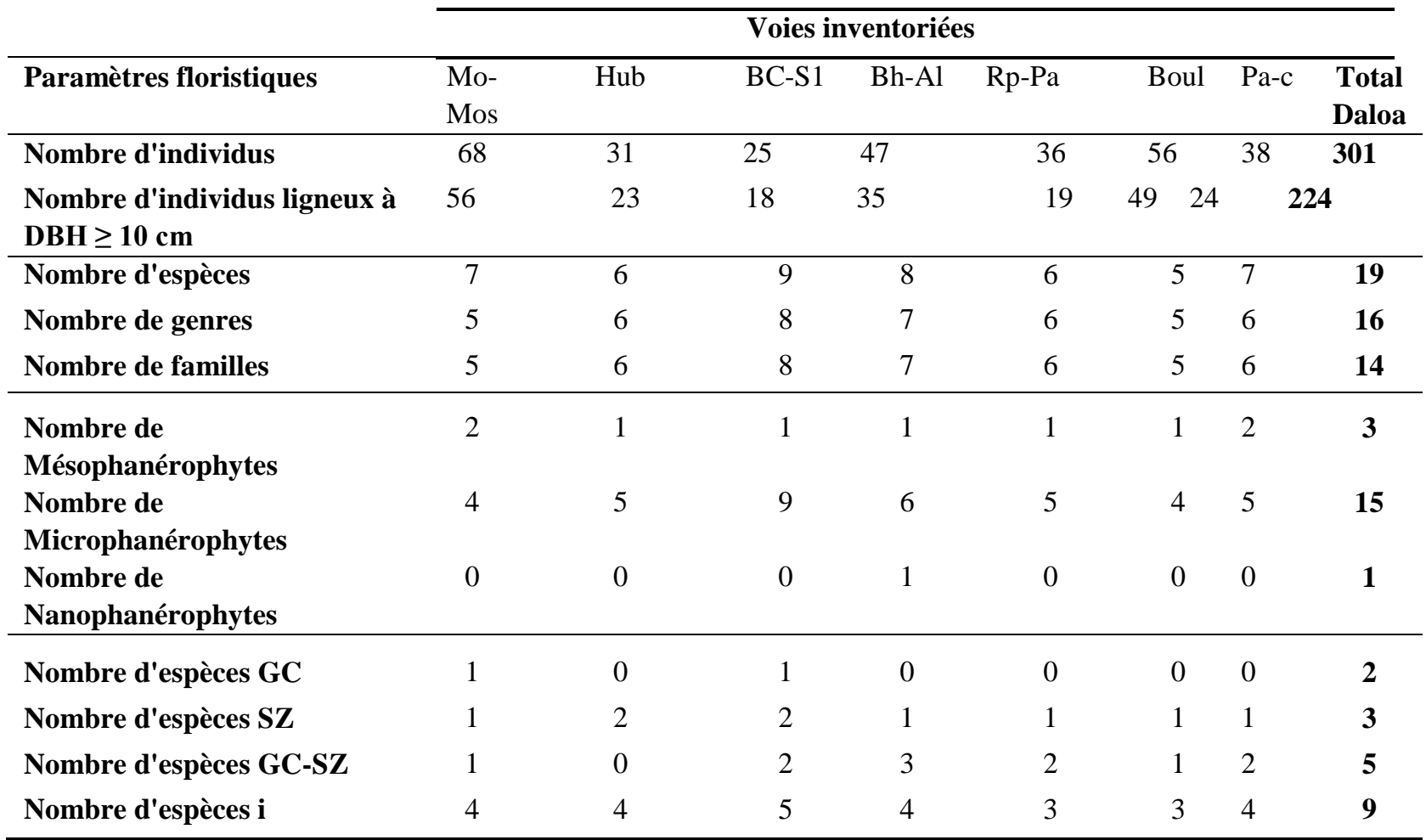

Mo : Monument ; Mos : Grande Mosquée ; Hub : Huberson goudron ; Bc : BCEAO ; S1 : Feu Soleil 1 ; Bh : BHCI ; Al : carrefour allocodrome ; Rp : Rondpoint ; Pa : carrefour pharmacie Parvis ; Boul : Boulevard ; Ac : carrefour Acémont.

Tableau 2: Similarité floristique entre les différentes voies étudiées.

\begin{tabular}{lccccccc}
\hline VOIES & Pa-Ac & Boul & Rp-Pa & Bh-Al & Bc-S1 & Hub & Mo-Mos \\
\hline Pa-Ac & 0 & 46,15 & 26,66 & 37,5 & 63,16 & 28,57 & 40 \\
Boul & & 0 & 66,67 & 30,77 & 37,5 & 18,18 & 33,33 \\
Rp-Pa & & & 0 & 26,67 & 33,33 & 30,77 & 42,86 \\
Bh-Al & & & & 0 & 52,63 & 28,57 & 40 \\
Bc-S1 & & & & & 0 & 35,29 & 44,44 \\
Hub & & & & & & 0 & 46,15 \\
Mo- & & & & & & & 0 \\
Mos & & & & & & & \\
\hline
\end{tabular}

Tableau 3: Distribution des tiges dans les classes de diamètres, nombre et pourcentage d'arbres, et surface terrière.

\begin{tabular}{ccc}
\hline Diamètre (cm) & Nombre d'individus et \% & Surface terrière $\left(\mathbf{m}^{\mathbf{2}}\right)$ \\
\hline$[10-20[$ & $59(26,34 \%)$ & 0,92 \\
{$[20-30[$} & $46(20,54 \%)$ & 2,11 \\
{$[30-40[$} & $8(3,57 \%)$ & 0,69 \\
{$[40-50[$} & $12(5,36 \%)$ & 2,02 \\
\hline
\end{tabular}




\begin{tabular}{ccc}
\hline$[50-60[$ & $30(13,39 \%)$ & 7,41 \\
{$[60-70[$} & $34(15,18 \%)$ & 11,36 \\
{$[70-80[$} & $25(11,16 \%)$ & 10,74 \\
{$[80-90[$} & $8(3,57 \%)$ & 4,65 \\
{$[90-100[$} & $2(0,89 \%)$ & 1,29 \\
Total & $\mathbf{2 2 4}$ & $\mathbf{4 1 , 1 9}$ \\
\hline
\end{tabular}

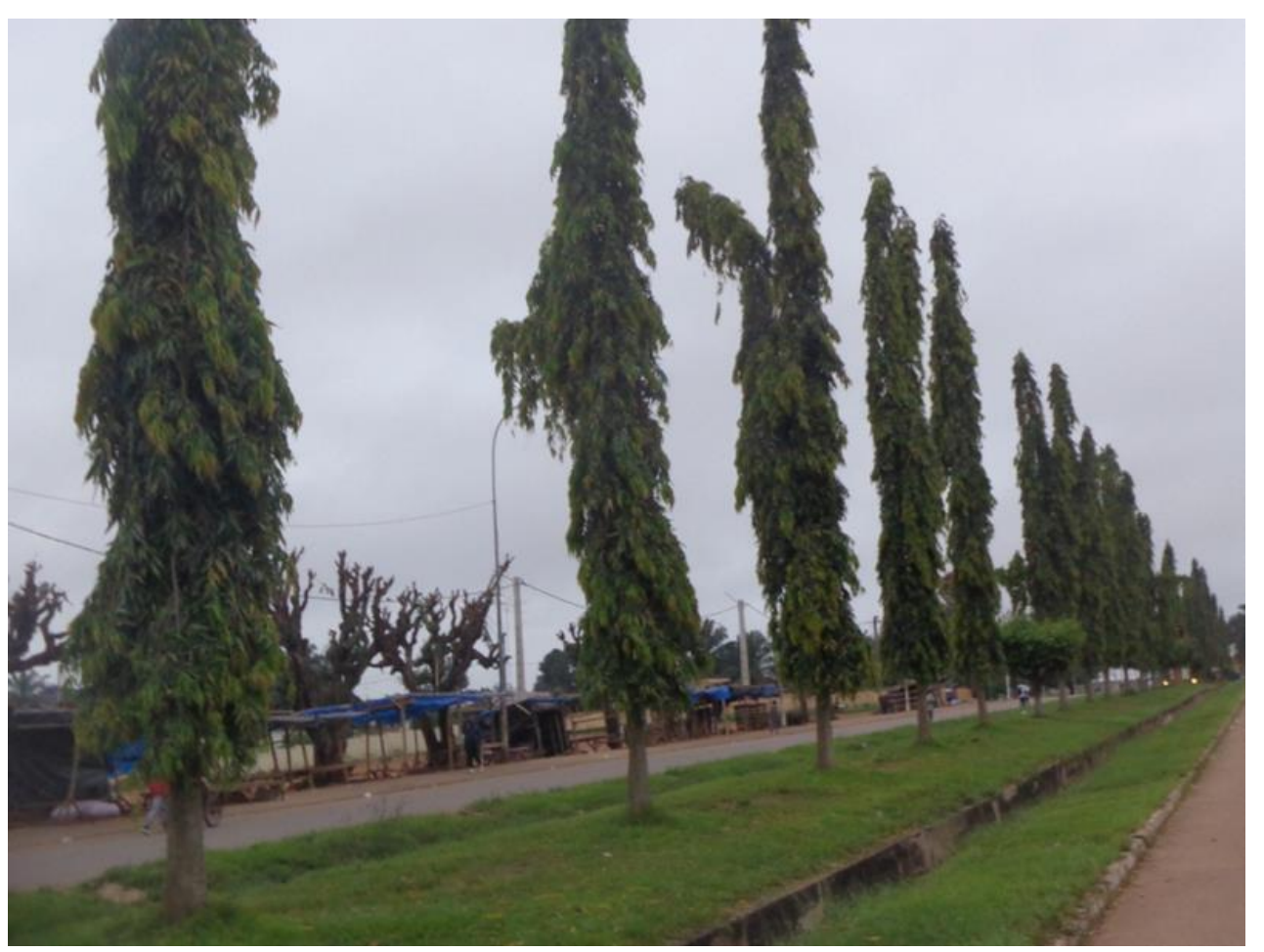

Figure 2 : Vue de Polyalthia longifolia en alignement sur le boulevard.

Tableau 4 : Paramètres structuraux et biomasse aérienne estimés sur les différentes voies.

\begin{tabular}{lcccccccc}
\hline & \multicolumn{9}{c}{ VOIES } & & & TOTAL \\
\hline & Pa-Ac & Boul & Rp-Pa & Bh-Al & Bc-S1 & Hub & Mo-Mos & $\mathbf{7}$ \\
\hline Longueur (Km) & 1,40 & 0,34 & 1,02 & 1,22 & 0,77 & 0,42 & 0,98 & 6,15 \\
Largeur (m) & 18 & 18 & 14 & 12 & 12 & 12 & 20 & 106 \\
Superficie (ha) & 2,52 & 0,612 & 1,428 & 1,464 & 0,924 & 0,504 & 1,96 & 9,41 \\
Densité (tiges/ha) & 9,52 & 80,06 & 13,30 & 23,90 & 19,48 & 45,63 & 28,57 & 220,46 \\
Biomasse aérienne (t) & 14,32 & 21,28 & 9,31 & 83 & 31,48 & 52,83 & 105,67 & 317,89 \\
Carbone séquestré (t) & 7,16 & 10,64 & 4,66 & 41,50 & 15,74 & 26,42 & 52,84 & 158,96 \\
CO $\mathbf{2}_{\mathbf{2}}$ équivalent (t) & 1,93 & 2,87 & 1,26 & 11,21 & 4,25 & 7,13 & 14,27 & 42,92 \\
\hline
\end{tabular}




\section{DISCUSSION}

\section{Richesse, composition et diversité des espèces recensées}

La présente étude révèle qu'il existe encore des arbres en alignement de voie dans la ville de Daloa. Cependant, nous avons remarqué que ces voies sont majoritairement bordées d'arbres alignés de façon discontinue. Aussi, ces arbres sont-ils le plus souvent disposés sur un seul côté. En effet, la ville semble avoir été pourvue autrefois d'arbre en alignement dans son plan d'aménagement. Mais ce type d'aménagement n'a pas résisté au temps. La faible richesse spécifique des arbres en alignement de voie constatée n'est pas spécifique à la ville de Daloa. Cette même remarque a été faite au niveau national sur les boulevards et avenues des communes du Plateau et de Cocody par Vroh et al. (2014) et Kouadio et al. (2016), au niveau africain par Gnele (2010) et au niveau mondial par Saribas (2008). Cette pauvreté des voies en arbres en alignement de la commune de Daloa pourrait s'expliquer par le fait que les questions environnementales n'occupent pas une place prioritaire dans les décisions politiques et stratégiques de nos élus locaux comme l'a souligné Ngahane (2015). En effet, la crise économique qui secoue les collectivités territoriales constitue une préoccupation majeure pour les gouvernants et la recherche de solutions à l'épineux problème de pauvreté. Cette quête perpétuelle de solutions les empêche d'envisager la protection de l'environnement gage du développement durable sur le long terme. A cela, s'ajoute également une population riveraine souvent peu voire mal renseignée sur les services et les fonctions des espaces verts urbains (Selmi, 2011) et les cas d'incivisme généralisé (Akionla, 2012). La forte présence de Ficus benjamina, Mangifera indica, Senna siamea, Azardirachta indica et Ficus polita en milieu urbain a déjà été signalé à Porto-Novo (au Benin). Cette dominance serait due à leurs fortes valeurs d'usage et à leur croissance rapide. En effet, selon Lougbégnon (2013), ces arbres sont couramment utilisés comme plantes ombragées, alimentaires, médicinales et ornementales en milieu urbain. De plus, leur taille moyenne leur permet de s'insérer aisément dans le tissu urbain sans encombrer la voie tout en participant activement aux bien être des citadins. Par ailleurs, Dardour et al. (2014) ont fait remarquer aussi que cette dominance est due au fait que la plupart de ces espèces s'adaptent bien aux conditions climatiques et édaphiques de la ville. Il est important de signaler que la forte proportion d'espèces introduites inventoriées témoigne de l'intérêt accordé à ces dernières dans les aménagements urbains aux dépens des espèces locales. En effet, la nature du tissu urbain influence la vision des acteurs si bien que la fonction esthétique et récréative des arbres et des espaces verts est ce qui leur confère le plus de valeur aux yeux des citadins et des visiteurs (DaCunha, 2009). Cette situation pourrait impacter négativement la survie des espèces locales en ville et la vie des populations locales urbaines qui utilisent souvent les différentes parties de ces espèces végétales pour leurs besoins quotidiens. Pour l'ensemble des voies étudiées, les valeurs élevées des différents indices de diversité montrent que diverses espèces sont utilisées en alignement des voies de la commune de Daloa. En effet, le reboisement est toujours guidé par le choix des espèces à reboiser et aussi par l'objectif du reboisement. Cette situation laisse la possibilité aux aménagistes d'associer des espèces en plantation d'alignement dans le but d'éviter les problèmes phytosanitaires liés à l'utilisation d'une seule selon Bekkouch et al. (2011). Cette politique de diversification est confirmée par la forte dissemblance floristique constatée entre les voies. Il est clairement établi que le planting d'arbre en alignement de voie n'est donc pas un fait hasard mais relève d'une action très raisonnée.

\section{Diversité structurale et principal rôle écologique des arbres}

La grande proportion des arbres de petits diamètres (]10-20 cm [et de ] 30-40 $\mathrm{cm}$ [) et la faible proportion des arbres de gros diamètres témoignent de la stabilité du milieu. En effet, la stabilité d'un milieu est assurée lorsque l'effectif des individus d'un 
peuplement forestier diminue régulièrement lorsqu'on passe d'arbres de petits DBH aux plus gros arbres. Cette situation serait due à un renouvèlement du peuplement arborescent des milieux. La politique de plantation d'arbres en alignement semble être une initiative très récente.

La faible densité en espèces végétales des voies, quant à elle, émane d'un phénomène global devenu classique dans les milieux urbains africains. En effet, les villes africaines sont caractérisées dans leur ensemble par un accroissement anarchique des surfaces construites (Nilson et al., 2000) au détriment du couvert végétal. Dans la plupart du temps, les travaux de recasement et d'ouverture/élargissement de voies ne font que renforcer cette dégradation (Amontcha et al., 2015). Ainsi, très souvent, les arbres d'alignement sont éliminés lors des travaux de reprofilage et de bitumage des voies. Cependant, la plus forte densité du boulevard obtenue dans cette étude est le résultat de la politique communale. En effet, de toutes les voies de la commune, elle est la seule qui a bénéficié d'un aménagement régulier. Le caractère très attrayant de ce boulevard a fait d'elle le lieu privilégié des habitants de la ville notamment les jeunes pour la pratique du sport.

Le taux de carbone séquestré par l'ensemble des arbres en alignement de voies de la ville de Daloa est de 42,92 tonnes. Ce faible taux de séquestration de carbone des arbres urbains est comme, l'ont souligné Dubé et al. (2006), la conséquence de la faible densité des forêts urbaines par rapport aux forêts naturelles. Cependant, malgré qu'elle soit de petite quantité, cette valeur revêt tout de même une importance capitale pour l'équilibre de l'écosystème urbain. En effet, cette quantité de carbone séquestrée contribuera à réduire significativement les effets de serre et le phénomène d'îlot de chaleur urbain.

\section{Conclusion}

L'étude des arbres en alignement des voies a permis de connaître la diversité et certains services écosystémiques fournis par ces arbres. En effet, la flore de l'étude est riche d'environ trois cent un (301) spécimens d'arbres. Parmi ceux-ci, deux cent vingtquatre (224) ont un DBH et une hauteur supérieure à $10 \mathrm{~cm}$ et $2 \mathrm{~m}$ respectivement. Ces espèces sont pour la plupart exotiques. Les voies inventoriées présentent une homogénéité et une dissemblance floristiques. La structure diamétrique des arbres révèle une discontinuité dans la distribution des arbres dans les différentes classes. Ces arbres séquestrent environ 42,92 tonnes de carbone et contribuent ainsi significativement à l'épuration de l'air et à la régulation du climat en milieu urbain. Avec la disparition des forêts naturelles, il devient donc indispensable de promouvoir la création des arbres en alignements de voie car leur taux de séquestration n'est pas négligeable. Cette action pourra contribuer efficacement à résoudre les nombreux problèmes écologiques auxquelles sont confrontés nos villes et leurs habitants.

\section{CONFLIT D'INTERETS}

Les auteurs déclarent qu'ils n'ont pas de conflit d'intérêts.

\section{CONTRIBUTIONS DES AUTEURS}

La conception de la méthodologie d'inventaire et du choix des sites d'étude ont été effectués sur la supervision de HRK. Les analyses statistiques et la correction du manuscrit ont été réalisées avec la collaboration de HKK. JKK est l'investigateur principal ; il s'est aussi assuré de la rédaction, de la discussion, de la mise en forme du document et des différentes corrections.

\section{REMERCIEMENTS}

Les auteurs de ce manuscrit tiennent à exprimer leurs profondes gratitudes et leurs vifs remerciements à toutes les autorités de la mairie de Daloa pour l'autorisation et l'appui technique dont nous avons bénéficiés. A tous nos référés qui ont pris de leur précieux temps pour parcourir minutieusement ce document dans toute sa genèse afin de le parfaire. Recevez ici chers Maîtres, notre grande admiration. Nous remercions enfin toutes les 
personnes qui ont contribué à la réalisation de ce travail.

\section{REFERENCES}

Akionla MA. 2012. Diversité et fonctions des formations végétales dans la ville de Porto-Novo. Mémoire de DESS en Gestion de l'Environnement, Université de Parakou, Bénin, 83 p.

Aguaron E, McPherson EG. 2012. Comparison of Methods for Estimating Carbon Dioxide Storage by Sacramento's Urban Forest. Urban Ecosystems and Social Dynamics Program, USDA Forest Service, 1731 Research Park Dr, Davis, CA 95618, USA ; 300 p.

Aké-Assi L. 2001. Flore de la Côte d'Ivoire 1, Catalogue, Systématique, Biogéographie et Ecologie. Conservatoire et Jardin Botanique : Genève, Suisse ; 396 p.

Aké-Assi L. 2002. Flore de la Côte d'Ivoire 2, Catalogue, Systématique, Biogéographie et Ecologie. Conservatoire et Jardin Botanique : Genève, Suisse ; 441 p.

Amontcha AAM, Lougbégnon T, Tente B, Djego J, Sinsin BA. 2015. Aménagements urbains et dégradation de la phytodiversité dans la Commune d'Abomey Calavi (Sud Bénin). Journal of Applied Biosciences, 91: 8519 - 8528. DOI: http://dx.doi.org/10.4314/jab.v91i1.9

Bekkouch I, Kouddane NE, Daroui EA, Boukroute A, Berrichi A. 2011. Inventaire des arbres d'alignement de la ville d'Oujda (Maroc). Revue "Nature \& Technologie »C- Sciences de l'Environnement, 05 : 87 - 91. DOI : www.univchlef.dz/revuenatec/art_05_11.

Da Cunha A. 2009. Introduction. In Urbanisme Végétal et Agriurbanisme, Da Cunha A (ed.). Observatoire Universitaire de la Ville et du Développement durable: Lausanne, Suisse ; p.20.

Dardour M, Daroui EA, Boukroute A, Kouddane NE, Abdelbasset B. 2014. Inventaire et état sanitaire des arbres d'alignement de la ville de Saïdia (Maroc oriental). Revue "Nature \& Technologie ». C-Sciences de l'Environnement, 10 : 02-09. DOI : www.univ chlef.dz/revuenatec/issue_10_art_c_01.

Dubé A, Saint-Laurent D, Senécal G. 2006. Penser le renouvellement et la politique de conservation de la forêt urbaine à l'ère du réchauffement climatique, p. 50.

Gnele JE. 2010. Dynamiques de planification urbaine et perspectives de développement durable à Cotonou (République du Bénin). Thèse de Doctorat unique EDP/FLASH, Université d'Abomey-Calavi (UAC), p. 338.

Guillaumet JL, Adjanohoun E. 1971. La végétation. In : le milieu naturel de la Côte d'Ivoire. Mémoire ORSTOM, 50, 157-263.

Kouadio YJC, Vroh BTA, Goné Bi ZB, Adou Yao CY, N'guessan KE. 2016. Evaluation de la diversité et estimation de la biomasse des arbres d'alignement des communes du Plateau et de Cocody (Abidjan - Côte d'Ivoire). Journal of Applied Biosciences, 97: 9141 - 9151. DOI: http://dx.doi.org/10.4314/jab.v97i1.1

Kouamé NN, N'guessan FK, N'guessan HA, N'guessan PW, Tano Y. 2015. Variations saisonnières des populations de mirides du cacaoyer dans la région du Haut-Sassandra en Côte d'Ivoire. Journal of Animal \& Plant Sciences, 25: (1) 3787-3798. DOI: http://www.m.elewa.org/JAPS/wpcontent/uploads/2015/05/1.ndri_.

Lougbégnon TO. 2013. Evaluation de la diversité des essences forestières urbaines de la ville de Porto-Novo et leurs utilisations par les populations locales. Revue de Géographie $d u$ Laboratoire Leïdi, 11 : 326-341. DOI : www.ugb.sn/revues

lsh/images/LEIDI/LEIDI11/Leidi1121Toussaint.

Malhi Y, Baker TM, Phillips OL, Almeida S, Alvarez E, Arroyo L. 2004. The aboveground coarse wood productivity of 104 
neotropical forest plots. Global Change Biology, 10:563-91. DOI: 10.1111/j. 1529-8817.2003.00778.x

McHale MR, Burke IC, Lefsky MA, Peper PJ, McPherson EG. 2009. Urban forest biomass estimates: is it important to use relationships developed specifi cally for urban trees? Urban Ecosyst, 12: 95-113. DOI 10.1007/s11252-009-0081-3.

Ngahane EL. 2015. Gestion technique de l'environnement d'une ville (Bembereke au Benin) : caractérisation et quantification des déchets solides émis ; connaissance des ressources en eau et approche technique. Thèse de Doctorat SGE, Université de Liège (Belgique), p. 239.

Nilson K, Randrup TB, Wandall BM. 2000. Trees in the urban environment. In The Forest Handbook. Blackwell Science 1: Oxford; 347-361.

Osseni AA, Mouhamadou IT, Tohozin BAC, Sinsin B. 2015. SIG et gestion des espaces verts dans la ville de Porto-Novo au Bénin. Tropicultura, 33 (2) : 146-156. DOI :

www.tropicultura.org/text/v33n2/146.pd $\mathrm{f}$

Polorigni B, Raoufou R, Kouami K. 2014. Perceptions, tendances et préférences en foresterie urbaine: cas de la ville de Lomé au Togo. European Scientific Journal, 10:(5) 261-277. DOI : http://dx.doi.org/10.19044/esj.2016.v12n 6 p168

Raunkiaer. 1934. The Life Forms of Plants and Statistical Geography. Claredon: Oxford; 632.
Reygrobellet B. 2007. La nature dans la ville: biodiversité et urbanisme. Etude du Conseil Economique et Social, les Editions des journaux officiels, 24, p.172.

Rondeux J. 1993. La Mesure des Arbres et des Peuplements Forestiers. Les Presses Agronomiques de Gembloux : Belgique ; 521.

Saribas M. 2008. Les plantations d'alignements en Turquie. Journal of Bartin Faculty of Forestry, 10(4): 50-56. DOI : htt://bof.karaelmas.edu.tr/journal.

Selmi W. 2011. Espace verts publics, entre planification urbaine et attentes des citadins. Laboratoire Image Ville Environnement, Strasbourg, $50 \mathrm{p}$.

Tra Bi ZA, Brou YT, Mahé G. 2015. Analyse par télédétection des conditions bioclimatiques de végétation dans la zone de contact forêt-savane de Côte d'Ivoire : cas du « V » Baoulé. XXVIIIe Colloque de l'Association Internationale de Climatologie, Liège (Belgique).

Vrain P. 2003. Ville durable et transports : automobile, environnement et comportements individuels. Innovations, Cahier d'Economie de l'Innovation, 18 : 91-112. Vroh BTA, Tiébré MS, N'Guessan KE. 2014. Diversité végétale urbaine et estimation du stock de carbone : cas de la commune du Plateau, Abidjan, Côte d'Ivoire. Afrique Science, 10(3) : 329- 340. DOI : https://www.ajol.info/index.php/afsci/art icle/download/109751/99499. 